\title{
Intracerebral microvascular measurements during deep brain stimulation implantation using laser doppler perfusion monitoring
}

Karin Wårdell, P. Blomstedt, Johan Richter, Johan Antonsson, Ola Eriksson, Peter Zsigmond, A.T. Bergenheim and M.I. Hariz

\section{Linköping University Post Print}

N.B.: When citing this work, cite the original article.

Original Publication:

Karin Wårdell, P. Blomstedt, Johan Richter, Johan Antonsson, Ola Eriksson, Peter Zsigmond, A.T. Bergenheim and M.I. Hariz, Intracerebral microvascular measurements during deep brain stimulation implantation using laser doppler perfusion monitoring, 2007, Stereotactic and Functional Neurosurgery, (85), 6, 279-286.

http://dx.doi.org/10.1159/000107360

Copyright: Karger

http://www.karger.com/

Postprint available at: Linköping University Electronic Press http://urn.kb.se/resolve?urn=urn:nbn:se:liu:diva-48444 


\title{
Intracerebral microvascular measurements during deep brain stimulation implantation using laser Doppler perfusion monitoring
}

\author{
Karin Wårdell ${ }^{1}$, Patric Blomstedt ${ }^{2}$, Johan Richter ${ }^{3}$, Johan Antonsson ${ }^{1}$, Ola Eriksson ${ }^{1,4}$,Peter \\ Zsigmond $^{4}$, A.Tommy Bergenheim ${ }^{2}$, Marwan. I. Hariz ${ }^{2,5}$ \\ ${ }^{1}$ Department of Biomedical Engineering, Linköping University, Sweden \\ ${ }^{2}$ Department of Neurosurgery, University Hospital, Umeå, Sweden \\ ${ }^{3}$ Department of Neurosurgery, University Hospital, Linköping, Sweden \\ ${ }^{4}$ Elekta Instrument AB, Stockholm, Sweden \\ ${ }^{5}$ Institute of Neurology, Queens Square, University College London, UK
}

Correspondence to:

Karin Wårdell

Department of Biomedical Engineering

Linköping University

S-581 85 Linköping

Email: karwa@imt.liu.se

Phone: +46-13-222455

Fax: +46-13-101902

Keywords: laser Doppler perfusion monitoring, deep brain stimulation, microcirculation, stereotactic neurosurgery

Short title: Laser Doppler perfusion monitoring during DBS-implantation 


\begin{abstract}
The aim of the study was to investigate if laser Doppler perfusion monitoring (LDPM) can be used in order to differentiate between gray and white matter and to what extent microvascular perfusion can be recorded in the deep brain structures during stereotactic neurosurgery. An optical probe constructed to fit in the Leksell ${ }^{\circledR}$ Stereotactic System was used for measurements along the trajectory and in the targets (GPi, STN, Zi, Thalamus) during the implantation of DBS-leads $(n=22)$. The total backscattered light intensity (TLI) reflecting the grayness of the tissue, and the microvascular perfusion was captured at 128 sites. Heartbeat-synchronized pulsations were found at all perfusion recordings. In six sites the perfusion was more than 6 times higher than the closest neighbor. TLI was significantly higher $(\mathrm{p}<0.005)$ and the perfusion significantly lower $(\mathrm{p}<0.005)$ in positions identified as white matter in the respective MRI-batch. The measurements imply that LDPM has the potential to be used as an intracerebral guidance tool.
\end{abstract}




\section{Introduction}

During intervention in the deep brain structures, by e.g. radio frequency (RF) lesioning [1], deep brain stimulation (DBS) [2] or neural cell grafting [3], safe, accurate and precise intracerebral navigation towards the pre-calculated target is imperative. Impedance methods can discriminate between gray matter, white matter and cerebrospinal fluid [4]. Physiological mapping using microelectrode recording (MER) or confirmation of anatomical targets using macro-stimulation are methods used to confirm targeting during stereotactic neurosurgery [5]. MER, however, may cause an increased risk of bleeding and does not constitute a guarantee for proper targeting [6],[7]. Furthermore, the MER signals may be difficult to interpret and may be misleading [8]. In order to overcome this, promising attempts have recently been made in order to introduce automatic signal processing algorithms and visualization of microelectrode recording signals during insertion of the electrodes towards the targets $[9,10]$.

One possible way to increase the precision, accuracy and safety in localizing the precalculated target during stereotactic procedures could be intracerebral recordings of optical signals. Giller and co-workers [11,12] presented a system using a probe with optical fibers for near-infrared intracranial measurements during stereotactic procedures in humans. By analyzing the slope of the reflected light spectra in the wavelength range $700-850 \mathrm{~nm}$ a separation between white and gray matter was possible. Our group recently showed that the same result is achieved using a fixed wavelength within the suggested spectral interval [13]. Thus, $780 \mathrm{~nm}$ is a commonly applicable wavelength in laser Doppler perfusion monitors (LDPM) which indicates that LDPM can be used to record not only microvascular blood perfusion but also tissue boundaries during stereotactic neurosurgery.

Laser Doppler perfusion monitoring [14] and imaging [15] are optical methods based on the detection of backscattered laser light from a small tissue volume containing both Dopplershifted and un-shifted scattered photons originating from the static tissue and the moving red blood cells. A perfusion value is defined as the concentration of moving red blood cells times their mean velocity and related to relative changes in the tissue's microcirculation, whereas the total backscattered light intensity (TLI) corresponds to the tissue's reflectivity at the laser wavelength used by the LDPM-system. Since the beginning of the 1980s, the laser Doppler 
technique has been used in a wide range of applications e.g. for assessment of skin reactions [16,17], burns [18] skin tumors [19], for the intra-operative monitoring of myocardial blood perfusion during bypass surgery [20] and during the evaluation of cortical brain microcirculation [21]. A review of the laser Doppler technique and its applications has been presented by Nilsson and co-workers [22].

In this study it is explored if LDPM can be used for measurements of tissue type and microcirculation in the deep brain structures during stereotactic neurosurgery in humans. The aim of the study was to investigate if a modified LDPM system could be used in order to differentiate between gray and white matter and to what extent microvascular perfusion could be recorded along the trajectory and in the target area.

\section{Material and Methods}

\section{Patient material}

Seventeen patients (seven women, age 40-72, mean \pm s.d. $=56 \pm 11$ ) referred for unilateral or bilateral DBS-implantation for the treatment of Parkinson's disease, essential tremor, dystonia or pain were included in the study. In total 22 leads were implanted. The study was approved by the local ethics committees at the University Hospitals in Linköping and Umeå (D. no. M182-04) and informed consent was received from the patients. Measurements were performed during implantations in the subthalamic nucleus (STN, $\mathrm{n}=11$ ), the globus pallidus internus ( $\mathrm{GPi}, \mathrm{n}=4)$, the caudal zona incerta $(\mathrm{Zi}, \mathrm{n}=2)$, and the thalamus $(\mathrm{Th}, \mathrm{n}=5$ : ventral intermediate nucleus [Vim, $n=2$ ]; ventral posterolateral nucleus [VPL, $n=2$ ]; ventral posteromedial nucleus [VPM, $\mathrm{n}=1$ ]. Eleven procedures took place at Umeå University Hospital and six at Linköping University Hospital.

\section{Laser Doppler system and measurement probe}

A system for intracerebral recordings of both microvascular perfusion and total backscattered light intensity (TLI) was set-up. It comprises a laser Doppler perfusion monitor (Periflux 5000 , Perimed AB, Sweden) a specially designed optical probe and a personal computer with software for acquisition, data analysis and presentation. The software, developed in Labview (National Instruments Inc., USA), made it possible to sample, store and present both the 
perfusion and TLI signals on-line. Methods for postprocessing of the captured data were developed in Matlab ${ }^{\circledR}$ (Mathworks Inc., USA). An overview of the system is presented in Figure 1.

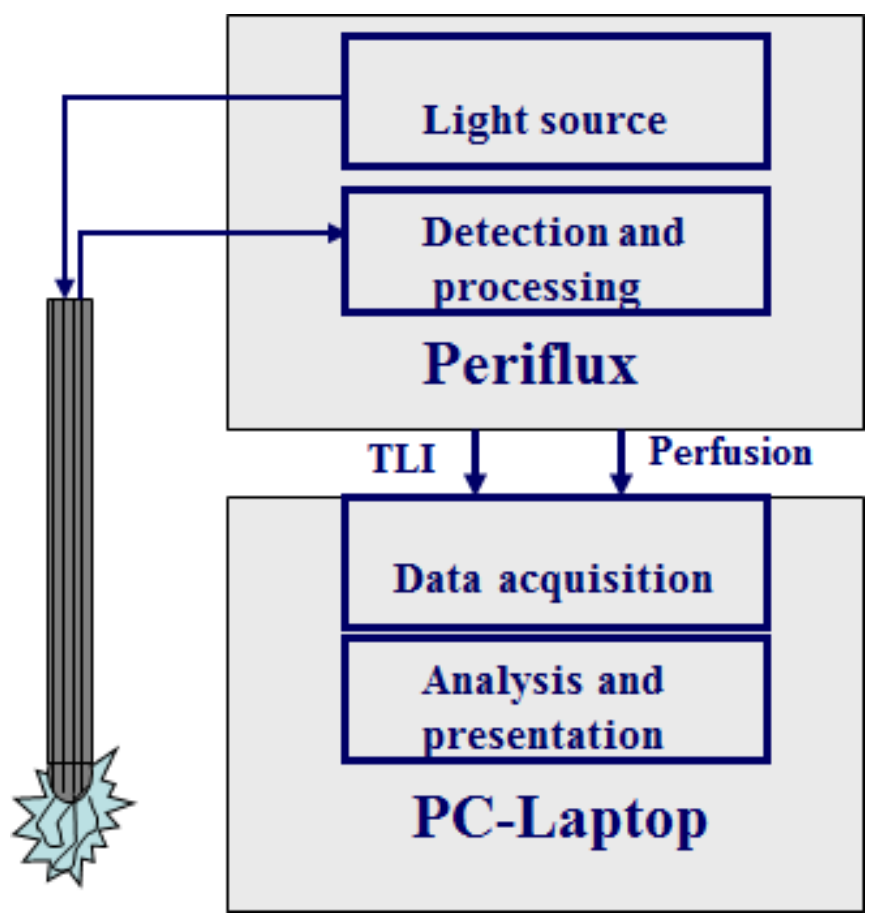

Figure 1. Overview of the laser Doppler perfusion monitoring system used for intracerebral measurements. The probe with fiber optics is connected to the light source and the detector unit in the Periflux. The TLI and perfusion signals are then sampled into a personal computer for processing and presentation.

The measurement probe was constructed with dimensions similar to a standard radiofrequency electrode. In order to fit in the Leksell ${ }^{\circledR}$ Stereotactic System (Elekta Instrument AB, Sweden) the probe's outer shaft was rigid, made of stainless steel and had a functional length of $190 \mathrm{~mm}$. The diameter was $2.2 \mathrm{~mm}$ except for the last $30 \mathrm{~mm}$ towards the tip where it was $1.5 \mathrm{~mm}$. Four optical fibers (step index, $\varnothing=240 \mu \mathrm{m}$ ) were aligned along the interior side of the probe towards the tip. Two of the fibers were used for laser Doppler recordings and two for reflection spectral measurements [13]. With this probe design, the tissue directly in front of the tip was investigated. 
The LDPM-system uses a low power, solid state laser $(1 \mathrm{~mW}, \lambda=780 \mathrm{~nm})$. During recording the laser light is guided through one of the optical fibers toward the tissue. After light interaction with the moving red blood cells in the tissue, backscattered, Doppler-broadened laser light is guided back through a second fiber to a detector unit in the Periflux. The light is processed to a value scaled linearly to tissue perfusion within a bandwidth of 0.02 to $12 \mathrm{kHz}$. In order to be able to capture fast perfusion changes, the time constant $(\tau)$ of the system was set to $0.03 \mathrm{~s}$. The total range of the perfusion and TLI signals was 0 - 999 arbitrary units (a.u.) and 0 - 10 a.u. respectively.

\section{Surgical technique and stereotactic imaging}

The surgical procedures differed slightly between the departments of neurosurgery in Umeå and Linköping. In general, the procedures were performed as described below. All procedures except for two cases of dystonia were performed under local anesthesia.

Stereotactic imaging was performed after placement of the Leksell ${ }^{\circledR}$ coordinate frame model G (Elekta Instrument AB, Sweden). The different targets in the thalamus were identified according to atlas-coordinates on stereotactic CT-studies. The target in the Vim was chosen 6-7 $\mathrm{mm}$ anterior to the posterior commissure (PC), at the level of the intercommissural line (ICL), and 13-15 mm lateral to the midline of the $3^{\text {rd }}$ ventricle. The target in the VPM and the VPL was 2-3 $\mathrm{mm}$ anterior to the PC, 2-3 $\mathrm{mm}$ below the ICL and $10 \mathrm{~mm}$ lateral to the midline of the $3^{\text {rd }}$ ventricle in VPM and $15 \mathrm{~mm}$ in the VPL.

Direct anatomical targeting was performed in the STN, GPi and Zi on stereotactic MRIstudies performed with a 1.5 Tesla scanner (Philips Intera, The Netherlands). Contiguous trans-axial slices of $2 \mathrm{~mm}$ thickness, T2-weighted sequences for STN and Zi and T1-weighted for GPi, were collected. The pallidal target was visually chosen $2 \mathrm{~mm}$ anterior to the midcommissural point, 2 -3 mm lateral of the pallido-capsular border on the axial slices, and about $2 \mathrm{~mm}$ above the optic tract on the coronal slices. The target in the STN was visually chosen at the line connecting the anterior borders of the nucleus Ruber, at the level of their maximal diameter, and approximately $1.5 \mathrm{~mm}$ lateral to the medial border of the STN. The depth was, when needed, corrected according to the lower border of the STN as seen on the 
coronal slices. The target in the caudal $\mathrm{Zi}$ was visually chosen slightly medial to the medial border of the STN, in the posterior part of the posterior third of the STN. The stereotactic images were exported to the Framelink Planning Station ${ }^{\circledR}$ (Medtronic, Minneapolis, MN, USA) or Leksell ${ }^{\circledR}$ Surgiplan (Elekta Instrument AB, Sweden) for calculation of target and trajectory.

The LDPM system was calibrated (motility standard, PF1001, Perimed AB, Sweden) prior to sterilization of the measurement probe. This certified a best-fit range of the system so both low and high perfusion values could be recorded in the same measurement session. To investigate the stability of the system a control measurement in motility was always carried out immediately after finalizing the measurement procedure.

At surgery a $14 \mathrm{~mm}$ burr-hole was placed according to the coordinates of the target allowing a trajectory avoiding penetration of the ventricles. Opening of the dura and a corticotomy were performed and the optical probe was introduced towards the target. During the introduction of the probe, measurements were performed at pre-designated points, starting at 40 or $30 \mathrm{~mm}$ from the target and continuing at 20,10,5 and $2.5 \mathrm{~mm}$ from the target as well as in the pre-calculated target area. Each recording lasted for 60 seconds, the total measurement session took about 15 minutes (in several procedures this also included diffuse reflection spectral measurements, see [13]). Two sequences also included cortex data and in two subjects the target measurement was omitted. In total 128 measurements where completed during insertion of the probe. Along two tracks one additional measurement was also obtained during withdrawal of the probe. During the recordings notes were taken of: recorded heart rate from the patient monitoring system, if the patient suffered from tremor or if other external interference with the recorded signal was present.

The optical probe was thereafter removed and replaced with the Medtronic DBS electrode $3387^{\circledR}$ or $3389^{\circledR}$ (Medtronic, Minneapolis, MN, USA) for macro-stimulation. The effect of intra-operative stimulation on symptoms such as tremor, rigidity, hypokinesia, and eventual induction of dyskinesias was evaluated and possible side effects, such as visual phenomena, capsular response, speech alterations and paresthesias were sought for. After achieving a 
satisfactory clinical response the electrode position was verified with a stereotactic CT and/or MRI, before implantation of the neurostimulator.

\section{Data analysis}

All measurement sequences were visually inspected and a 30 seconds section was selected from each recording for analysis of the perfusion and the TLI. Sections considered as external noise caused by e.g. known fiber movement artifacts or over-bending of the fiber were excluded in this selection. From the perfusion-signal: mean (m), standard deviation (s.d.), peak-to-peak (p-p) and heart rate (HR) were calculated. Furthermore the signal was studied regarding vasomotion and other types of possibly temporal variations caused by the microcirculation. For each TLI-sequence, the mean and standard deviation were calculated and if applicable the peak-to-peak. In order to identify the tissue type at the measurement site, inspection of pre-operative MRI was done using the surgical planning systems. The identified tissue was graded as white matter, gray matter or mixed. The mixed group contained positions where no clear classification could be made. Along each trajectory, the TLI and perfusion at the corresponding white and gray tissue sites were averaged. The identified data were then grouped both according to white and gray tissue and the brain target aimed at. Data were tested using the Wilcoxon paired signed rank test or the Mann Whitney U-test for grouped samples. $\mathrm{P}$ values $<0.05$ were considered significant.

\section{Results}

During all 22 DBS-implantations, both the microvascular perfusion and total backscattered light intensity were easily recorded and displayed on-line with the designed system and probe. Post-operative measurements in motility showed that the TLI signal was stable and varied less than $1.3 \%$. The calibration procedure certified that the perfusion signals were comparable from time-to-time.

An example of a trajectory with the measurement sites towards GPi superimposed on the Schaltenbrand-Wharen $+2 \mathrm{~mm}$ coronal image is illustrated in Fig. 2a. The corresponding perfusion and TLI measurement sequences for respective sites are presented in Fig. 2b-c. Pulsative variations in the perfusion signal agreed with the monitored heart rate. They were 
visible in all captured perfusion signals, but with varying peak-to-peak. In Fig. 2d-e, perfusion- and TLI averaged over 30 seconds are presented. The perfusion increased towards the target whereas the TLI presented an inverse relationship with higher values at 30 and 20 mm. Recordings towards STN are exemplified on a patient in Fig. 3. Along this trajectory, superimposed on the $-3 \mathrm{~mm}$ coronal image, elevated perfusion with a high peak-to-peak was found $5 \mathrm{~mm}$ from the target. In addition, the perfusion signal demonstrated a pronounced vasomotion pattern with 6 cycles/min. superimposed on the pulsations originating from the heartbeat (Fig. 3b). The existence of a blood vessel close to the measurement site was confirmed during post-operative image inspection of both the coronal and axial T2-weighted MRI. Average perfusion and TLI data related to respective measurement position are presented in Fig. 3c-d.

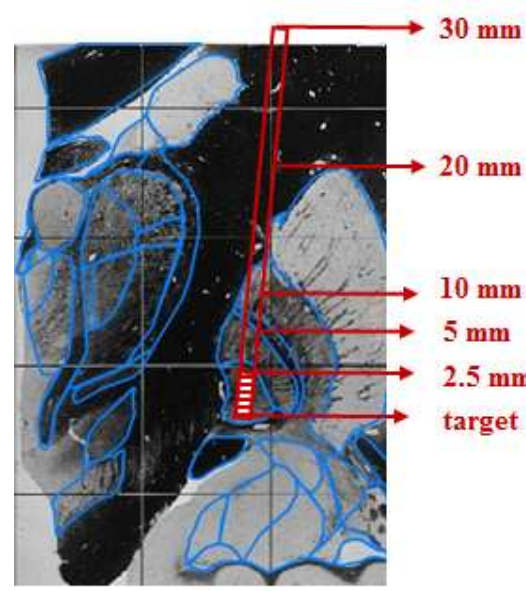

a

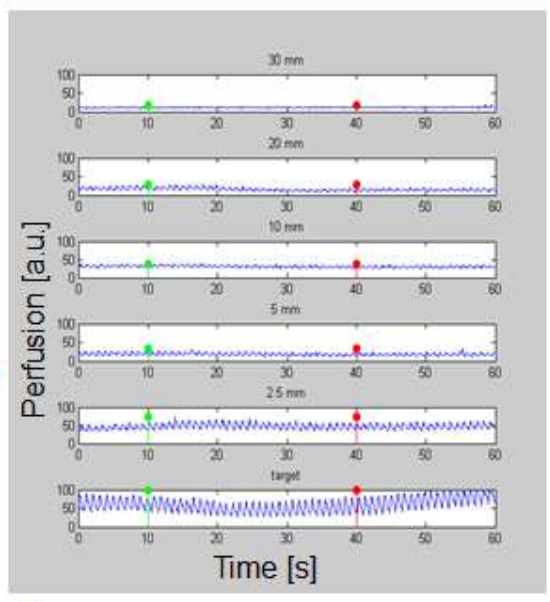

b

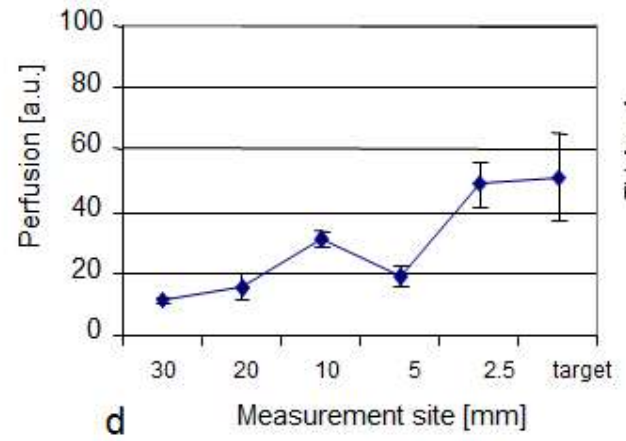

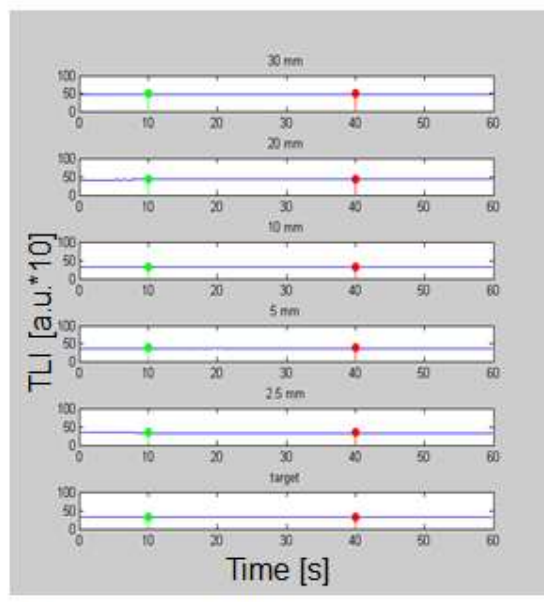

C

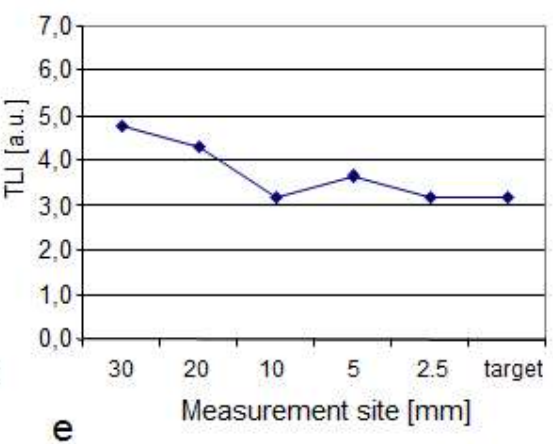

Figure 2. Example of perfusion and TLI signals captured from a typical measurement towards GPi: a) Probe trajectory and measurement sites superimposed on the Schaltenbrand-Wharen atlas, $+2 \mathrm{~mm}$ coronal image [27]. b) Time traces of perfusion, the pulsative variations correspond to the monitored heartbeat. c) Time traces of the TLI. $\mathrm{d}$ ) Averaged perfusion ( $\mathrm{m} \pm$ s.d.) plotted against measurement site. e) Averaged TLI (m) plotted against the measurement site. 


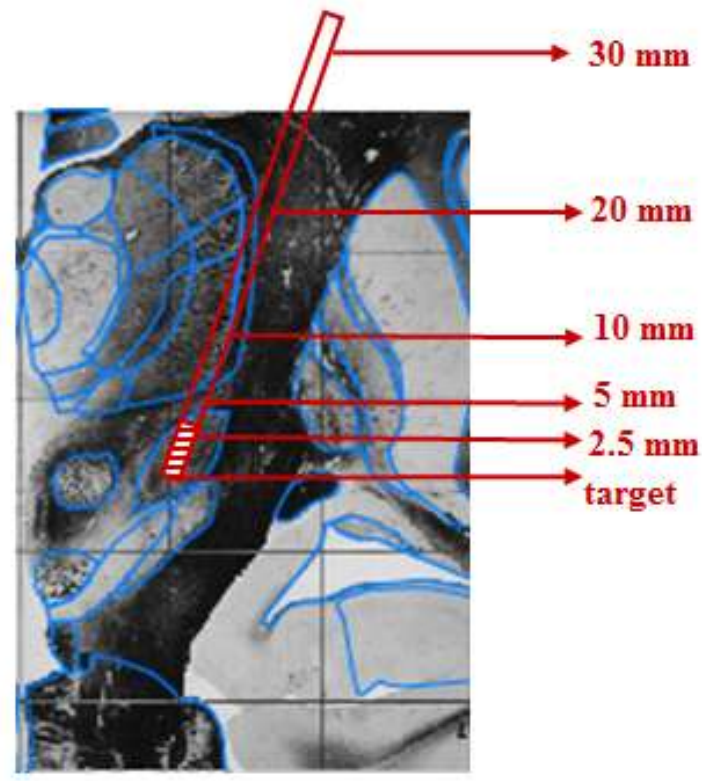

a

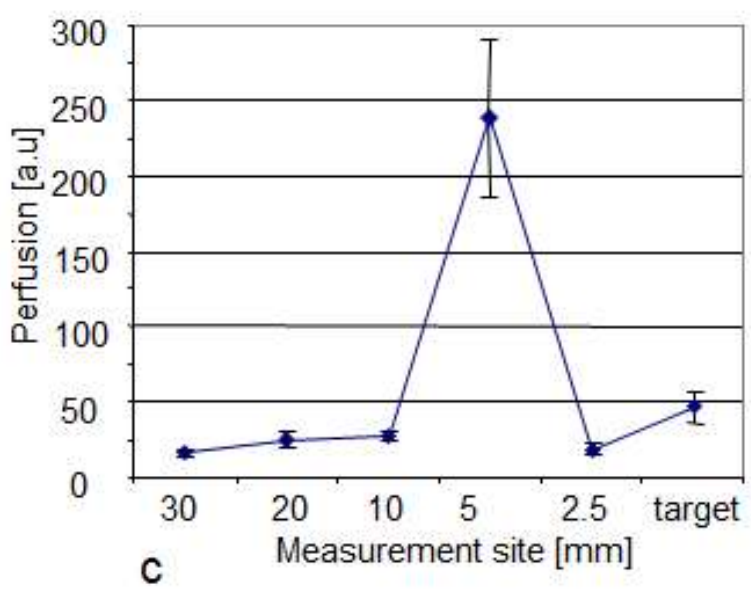

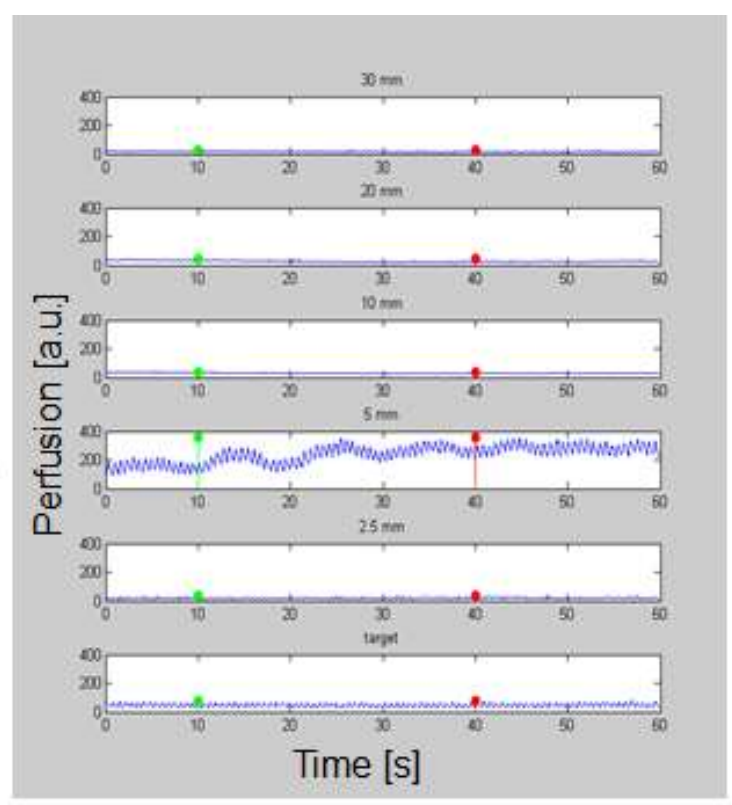

b

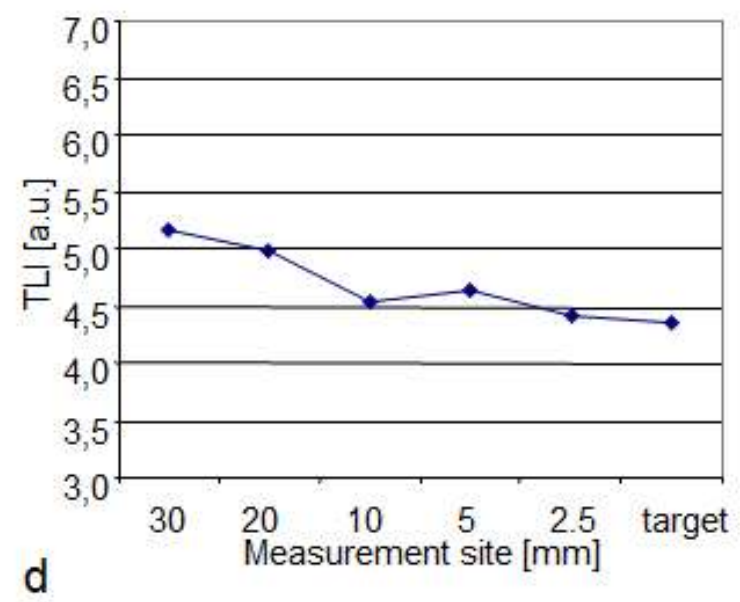

Figure 3. Example of a recording towards the STN. a) Probe trajectory and measurement sites are superimposed on the Schaltenbrand-Wharen atlas, $-3 \mathrm{~mm}$ coronal image [27]. b) Time traces of the perfusion, a highly pulsative perfusion signal was found $5 \mathrm{~mm}$ from the target. Heartbeat-related pulsations are superimposed on a 6 cycle/min. vasomotion pattern. c) Perfusion ( $\mathrm{m} \pm$ s.d.) and $\mathrm{d})$ TLI $(\mathrm{m})$ in relation to the measurement sites.

A summary of all the microvascular perfusion measurements $(n=128)$ recorded when the probe was inserted, and grouped according to target aimed at, is presented in Fig. 4a-c (GPi, STN, Zi) and Fig. 5a (Th). All presented values represent the average value from a registration covering 30 seconds. In six out of 128 measurement positions the perfusion was more than six times higher than at least one of the closest neighbors (marked with circles in 
Fig. 4 a-b and Fig. 5a, Tab. 1). The average perfusion for these high perfusion spots was $309.8 \pm 166.0$ a.u. When removing these "outliers", the average microvascular perfusion of all remaining recordings was $30.7 \pm 18.4$ a.u. $(\mathrm{n}=122)$.
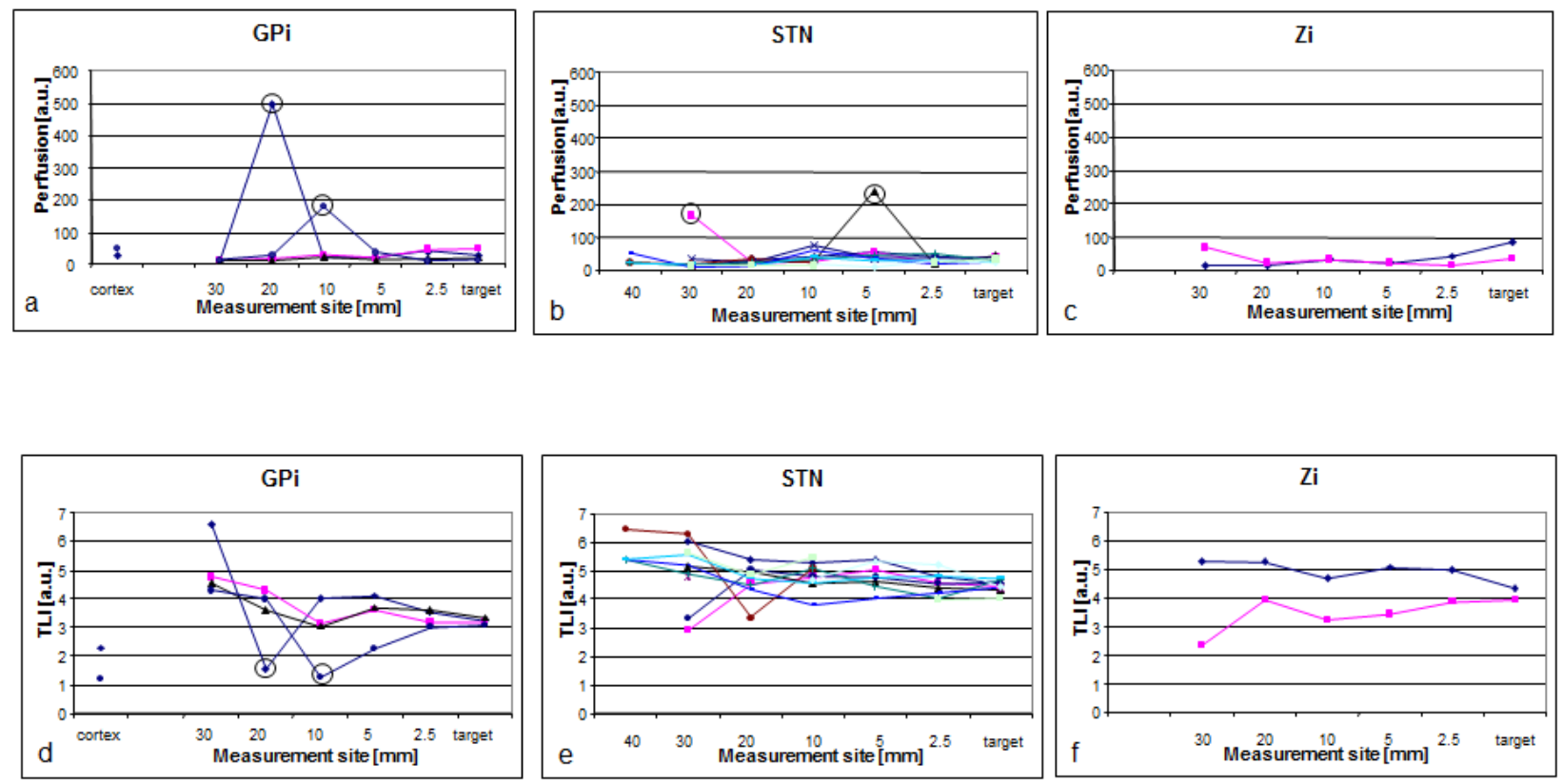

Figure 4. Summary of perfusion (a-c) and TLI (d-f) recordings grouped according to target aimed at (GPi, STN, Zi). High perfusion spots and very low TLI in relation to high perfusion spots are marked with circles.

A summary of all captured TLI grouped according to target aimed at, is presented in Fig. 4d-f (GPi, STN, Zi) and Fig. 5b (Th). In general the curves started with elevated TLI and leveled out towards the target. Two of the five lowest TLI values were recorded in the cortex and three in sites related to high perfusion spots (marked with circles in Fig. 4d, 5b). A peak-topeak in the TLI, with a frequency corresponding to the heartbeat, was found at two of the high perfusion spots (Tab. 1).

Along ten of the trajectories it was possible to identify measurement positions above the target as both white and gray tissue from respective MRI-stack. The TLI was significantly higher $(\mathrm{p}<0.005)$ and the perfusion significant lower $(\mathrm{p}<0.005)$ in positions identified as white tissue. The targets in GPi had a significant lower TLI than the STN ( $<<0.005)$. There was, however, no significant difference in perfusion between GPi and STN. 

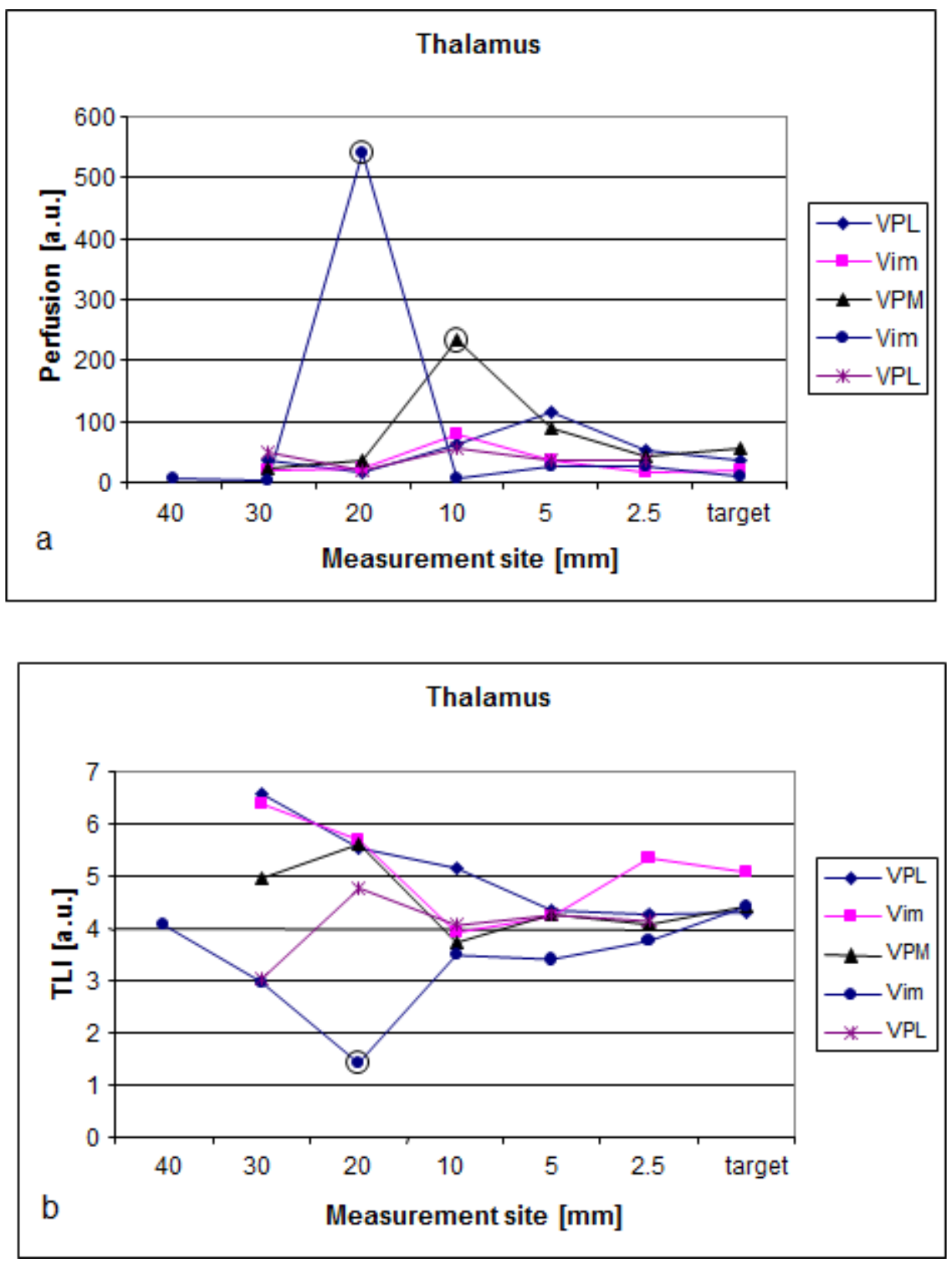

Figure 5. Summary of perfusion (a) and TLI (b) recordings along the trajectories towards targets in the thalamus (VPL, VPM, Vim). High perfusion spots and very low TLI in relation to high perfusion spots are marked with circles.

\section{Discussion}

In this study, laser Doppler perfusion data captured along pre-calculated trajectories towards individual nuclei in the deep brain structures have been presented. Compared to other intracerebral methods the LDPM-technique has the added advantage of recording not only the tissue's total backscattered light intensity reflecting the tissue's grayness, but also the 
microcirculation in the vicinity of the probe tip. This implies that the technique has a potential to detect not only gray-white boundaries but also increased blood flow along the trajectory.

Table 1. Measurement sites along the trajectory with elevated perfusion compared to surrounding sites. Two of the recordings were repeated during withdrawal of the probe.

\begin{tabular}{|c|c|c|c|c|}
\hline Target, Position & \multicolumn{2}{|c|}{$\begin{array}{l}\text { Perfusion [a.u.] } \\
\text { mean } \pm \text { s.d. p-p }\end{array}$} & \multicolumn{2}{|c|}{$\begin{array}{l}\text { TLI [a.u.] } \\
\text { mean } \quad \text { p-p }\end{array}$} \\
\hline $\mathrm{GPi}, 20 \mathrm{~mm}$ & $498 \pm 123$ & 417 & 1.6 & 0.4 \\
\hline repeated measurement & $228 \pm 24$ & 130 & 0.7 & 0.1 \\
\hline $\mathrm{GPi}, 10 \mathrm{~mm}$ & $179 \pm 27$ & 130 & 1.3 & - \\
\hline $\mathrm{STN}, 30 \mathrm{~mm}$ & $167 \pm 9$ & 52 & 3.0 & - \\
\hline $\mathrm{STN}, 5 \mathrm{~mm}$ & $238 \pm 53$ & 125 & 4.6 & - \\
\hline VPM, $10 \mathrm{~mm}$ & $234 \pm 11$ & 74 & 3.7 & - \\
\hline Vim, $20 \mathrm{~mm}$ & $542 \pm 86$ & 345 & 1.4 & 0.4 \\
\hline repeated measurement & $235 \pm 17$ & 88 & 1.0 & 0.3 \\
\hline
\end{tabular}

High pulsative microvascular perfusion was registered at six out of 128 measurement sites (Fig. 4a-b, Fig. 5a, Tab. 1). In two of these (20 $\mathrm{mm}$ from GPi and Vim respectively) the signal was more than 25 times higher than at the surrounding measurement positions. Repeated recordings when the probe was withdrawn confirmed the elevated perfusion, however, with reduced values. This is probably caused by a slightly different sampling volume surrounding the probe tip. When the probe is inserted the tissue in front of the tip is investigated, whereas when it is removed the tissue is disrupted. Along one of the STNtrajectories a highly elevated perfusion with a pronounced vasomotion pattern superimposed 
on the heartbeat was registered. Post-operative image inspection revealed the existence of a very small vessel in the vicinity of the probe. It is very likely that also the remaining recordings related to high perfusion were captured close to a similar vessel structure, however, not visible with the used MRI-protocol. Several of the high perfusion spots were accompanied by a lower TLI than could be expected (Fig. 4d, 5b, Tab. 1). These low TLIs were most likely caused by the increased absorption from blood. While two of these low TLI also had a heartbeat-synchronized variation it may be an additional indication that the signals were captured close to a vessel's structure. This was, however, not consistently valid for all high perfusion spots. By introducing 3T-MRI-scans together with Gadolinium as contrast medium it may be possible to elucidate the relationship between high perfusion spots along the trajectories, and closely related vessel structures.

In general the TLI was higher at the 40 and $30 \mathrm{~mm}$ sites compared to in the target area, indicating white tissue. Statistical analysis between TLI captured in white and gray tissue based on MRI was, however, only possible for 10 out of 17 trajectories. The reduced number of samples for comparison was caused by difficulty in judging the tissue type from the MRI at the pre-selected measurement positions, and several sites were therefore graded as mixed. Despite this, a significant difference between white and gray matter was found. This is in agreement with the reflection spectral measurements performed by Antonsson et al., [13]. In this study, different spectral signatures and intensities were found for gray and white matter. A high correlation $(\mathrm{r}=0.99, \mathrm{p}<0.0001, \mathrm{n}=78)$ was found between the tissue's reflectivity at the wavelength $780 \mathrm{~nm}$ (the LDPM wavelength) and spectral content along a slope ranging from 750-800 nm. Giller and colleagues [11,12] have also presented reflection spectra captured during stereotactic neurosurgery with the ability to separate between white, and in their case, cortex-gray matter, within the same wavelength interval.

With the used set-up, the LDPM signals were only registered at fixed pre-designated positions along the trajectories and several high perfusion spots could have been missed. In future studies the resolution along the trajectory can be increased by recording the perfusion and TLI at e.g. mm-distances. For the TLI it might even be possible to perform continuous measurements during insertion of the electrode and thus increase the spatial resolution and the 
possibility to discriminate between gray-white boundaries. One major drawback is, however, that the perfusion signal will be affected by the external movements generated by the probe insertion. When the movements of the red blood cells in the tissue are detected with the laser Doppler technique it is important to minimize all external movement influences on the signal. These can also be caused by the patient's involuntary movements or by touching, or overbending the fiber during a recording. At stereotactic neurosurgery using a frame-based system, both the patient's head and the measurement probe are fixated to the stereotactic system. Therefore not even major tremor showed any interference with the perfusion signal when recordings were made at fixed positions.

Another aspect that must to be accounted for is that only relative perfusion and TLI changes can be recorded. In the current study, however, the system was always calibrated and therefore all captured values were comparable. Furthermore, if the electrode's design changes, this can result in a different measurement range. A slightly different probe design can, for example, change the optical sampling depth, which is dependent not only on the tissue's scattering and absorption properties but also on the fiber separation distance in the probe and the used wavelength. Previous investigations using Monte Carlo simulations and experimental studies have shown that the optical sampling depth in brain tissue is about $1 \mathrm{~mm}$ [23-26].

In conclusion, this study shows the first attempt at using laser Doppler perfusion monitoring for intracerebral measurements during stereotactic neurosurgery. The technique can record both the microvascular changes along the trajectory as well as differentiate between gray and white matter. Future studies are needed in order to elucidate to what extent a gray-white discrimination can be achieved as well as to study the relationship between high perfusion spots and vessel structures. Furthermore, there is a need to include a comparison not only with MRI but also with established guidance methods such as microelectrode recording and impedance measurements in order to investigate to what extent LDPM can be used as an intracerebral guidance tool.

\section{Acknowledgements}


The authors wish to express their sincere gratitude to Carina Fors, M.Sc. and to research engineer Per Sveider, Dept. of Biomedical Engineering for skilful Labview programming and probe construction respectively, and to the staff at the MRI-units at Umeå and Linköping University Hospitals. The study was supported by the Vinnova-funded Swedish Competence Centre NIMED.

\section{References}

1. Laitinen LV, Bergenheim AT, Hariz MI: Leksell's posteroventral pallidotomy in the treatment of Parkinson's disease. J Neurosurg 1992;76:53-61.

2. Benabid AL: Deep brain stimulation for Parkinson's disease. Current Opinion in Neurobiology 2003;13:696-706.

3. Rehncrona S: A critical review of the current status and possible developments in brain transplantation. Adv Tech Stand Neurosurg 1997;23:3-46.

4. Laitinen LV, Hariz MI: Movement Disorders. in Youmans JR (ed): Neurological Surgery. Philadelphia: Saunders, 1996, 3575-3609.

5. Gross RE, Krack P, Rodriguez-Oroz MC, Rezai AR, Benabid AL: Electrophysiological mapping for the implantation of deep brain stimulators for Parkinson's disease and tremor. Mov Disord 2006;21:S259-S283.

6. Hariz MI: Safety and risk of microelectrode recording in surgery for movement disorders. Stereotact Funct Neurosurg 2002;78:146-157.

7. Palur RS, Berk C, Schulzer M, Honey CR: A metaanalysis comparing the results of pallidotomy performed using microelectrode recording or macroelectrode stimulation. J Neurosurg 2002;96:1058-1062.

8. Schiff SJ, Dunagan BK, Worth RM: Failure of single-unit neuronal activity to differentiate globus pallidus internus and externus in Parkinson disease. J Neurosurg 2002;97:119-128.

9. Falkenberg JH, McNames J, Burchiel KJ: Automatic microelectrode recording analysis and visualization of the globus pallidus interna and stereotactic trajectory. Stereotact Funct Neurosurg 2006;84:28-34.

10. Falkenberg JH, McNames J, Favre J, Burchiel KJ: Automatic analysis and visualization of microelectrode recording trajectories to the subthalamic nucleus: preliminary results. Stereotact Funct Neurosurg 2006;84:35-45.

11. Johns M, Giller CA, Liu HL: Computational and in vivo investigation of optical reflectance from human brain to assist neurosurgery. Journal of Biomedical Optics 1998;3:437-445.

12. Giller CA, Johns M, Liu H: Use of an intracranial near-infrared probe for localization during stereotactic surgery for movement disorders. J Neurosurg 2000;93:498-505.

13. Antonsson J, Eriksson O, Blomstedt P, Bergenheim AT, Hariz MI, Richter J, Zsigmond $\mathrm{P}$, Wårdell K: Diffuse reflectance spectroscopy measurements for tissue type discrimination during deep brain stimulation. unpublished data 2007.

14. Nilsson GE, Tenland T, Öberg PA: Evaluation of a laser Doppler flowmeter for measurement of tissue blood flow. IEEE Trans Biomed Eng 1980;27:597-604. 
15. Wårdell K, Jakobsson A, Nilsson GE: Laser Doppler perfusion imaging by dynamic light scattering. IEEE Trans Biomed Eng 1993;40:309-316.

16. Wårdell K, Naver HK, Nilsson GE, Wallin BG: The cutaneous vascular axon reflex in humans characterized by laser Doppler perfusion imaging. J Physiol 1993;460:185199.

17. Fullerton A, Stücker M, Wilhelm KP, Wårdell K, Anderson C, Fischer T, Nilsson GE, Serup J: Guidelines for visualization of cutaneous blood flow by laser Doppler perfusion imaging. A report from the Standardization Group of the European Society of Contact Dermatitis based upon the HIRELADO European community project. Contact Dermatitis 2002;46:129-140.

18. La Hei ER, Holland AJ, Martin HC: Laser Doppler imaging of paediatric burns: burn wound outcome can be predicted independent of clinical examination. Burns 2006;32:550-553.

19. Enejder AM, af Klinteberg C, Wang I, Andersson-Engels S, Bendsoe N, Svanberg S, Svanberg K: Blood perfusion studies on basal cell carcinomas in conjunction with photodynamic therapy and cryotherapy employing laser-Doppler perfusion imaging. Acta Derm Venereol 2000;80:19-23.

20. Karlsson MG, Fors C, Wårdell K, Casimir-Ahn H: Myocardial perfusion monitoring during coronary artery bypass using an electrocardiogram-triggered laser Doppler technique. Med Biol Eng Comput 2005;43:582-588.

21. Fasano VA, Urciuoli R, Bolognese P, Mostert M: Intraoperative use of laser Doppler in the study of cerebral microvascular circulation. Acta Neurochirurgica 1988;95:4048.

22. Nilsson GE, Salerud GE, Strömberg NOT, Wårdell K: Laser Doppler perfusion monitoring and imaging. In Biomedical Photonics Handbook 2003:Chapter 15, 11-24.

23. Jacobsson A: Sampling depth in laser Doppler flowmetry. In Lic. Eng. Thesis No. 307. Linköping University, 1992.

24. Larsson M, Steenbergen W, Strömberg T: Influence of optical properties and fiber separation on laser doppler flowmetry. J Biomed Opt 2002;7:236-243.

25. Giller CA, Liu HL, Gurnani P, Victor S, Yasdani U, German DC: Validation of a near-infrared probe for detection of thin intracranial white matter structures. $\mathbf{J}$ Neurosurg 2003;98:1299-1306.

26. Qian Z, Sunder SV, Yeqing G, Giller CA, Liu HL: "Look-ahead distance" of a fiber probe used to assist neurosurgery: Phanton and Monte Carlo study. Optics express 2003;11:1844-1855.

27. Nowinski WL, Thirunavuukarasuu M: The Cerify Clinical Brain Atlas on CD-ROM. Thieme Verlag 2004. 